\title{
SUPPLY CHAIN RISKS ANALYSIS BY USING JUMP-DIFFUSION MODEL
}

\author{
Xianzhe Chen \\ Dept. of Transportation and Logistics \\ $161612^{\text {th }}$ Avenue North, North Dakota State University \\ Fargo, ND 58105, USA
}

\begin{abstract}
This paper investigates the effects of demand risk on the performance of supply chain in continuous time setting. The inventory level has been modeled as a jump-diffusion process and the two-number inventory policy has been implemented in the supply chain system. The simulated annealing algorithm has been used to search the optimal critical values of the two-number policy. The jump magnitude has been considered in two cases: constant and Laplace distribution which has favorable property, i.e. leptokurtic. Numerical studies have been conducted for various scenarios to provide insights of effects of demand disruption on the performance of the supply chain.
\end{abstract}

\section{INTRODUCTION}

This paper investigates the effects of supply chain demand risk on the performance of the supply chain, in which the inventory level has been modeled by a jump-diffusion model. The model is based on continuous setting, which has little relevance to the inventory in which the individual items are physically or economically significant, but it still fits for high-volume discrete system flow (Harrison 1985).

In this paper we define the risk in two main parts: inherent risk and exogenous risk. The inherent risk is referred to the organizational intrinsic uncertainty, such as uncertain lead time. While the exogenous risk is referred as natural or man-made disruption, such as earthquake, tsunami, war and terrorism. The main objective of this paper is to investigate the effects of exogenous risk on the performance of supply chain.

The idea of using jump-diffusion model into supply chain disruption is that the inventory level can be thought of two components: one is the small continuous changes due to the normal balance dynamics between supply and demand, which is the counterpart of white noise in discrete time setting, and the other is the occasional jumps resulting from the release of new, significant information. The Wiener process can be used to represent the inherent supply
Jun Zhang

Dept. of Industrial and Manufacturing Engineering

$130112^{\text {th }}$ Avenue North, North Dakota State University

Fargo, ND 58105, USA

chain risk on demand side; on the other hand, the jump process denotes the exogenous risk of supply chain.

It is widely known that the supply chain risks will affect the performance of the supply chain, but so far there is no numerical study to exhibit how much effect it will cause. Therefore, it motivates us to conduct a simulation study to examine this phenomenon starting from a simple one-echelon supply chain. Hence, the primary purpose of this paper is to investigate the effects of supply chain risks on the performance of the supply chain in the form of longterm average cost and provide some managerial insights through numerical study. And we propose simulated annealing algorithm to search the optimal critical values in order to reduce the long-term average cost of the supply chain.

Continuous-time two-number inventory policy $\left(\mathrm{x}_{0}, \mathrm{x}_{1}\right)$ has been implemented and proved to be optimal in Vickson (1986) for a diffusion model. The mechanism of a twonumber inventory policy $\left(\mathrm{x}_{0}, \mathrm{x}_{1}\right)$ is as follows: when the inventory level $x(t) \leq x_{0}$, a machine which is off at time $\mathrm{t}$ will be turned on; while when the inventory level $x(t) \geq x_{1}$, a machine which is on at time $t$ will be turned off. If a machine switching occurs, it will lead to switching cost. This simply inventory policy has also been proved to be optimal in the case of discrete time (Graves and Keilson 1981).

The disruption could occur at supply side or demand side or both sides in a supply chain. An example for demand side is the effect of news on product demand, i.e. it is reported that consumption of the product prevents heart disease (or causes cancer) and the sales of the product would suddenly jump upward (or downward). And the disruption can also happen on supply side (Chopra and Sodhi 2004). Hendricks and Singhal (2005) investigates the longterm stock price effects of supply chain disruptions. It appears that the average abnormal stock returns of firms which experienced disruptions is nearly $-40 \%$. Hence, the supply chain disruption could significantly affect the production, schedule, and the financial performance of the company. Here we mainly investigate risks occurred at demand side and only consider one-echelon supply chain. 


\section{Chen and Zhang}

This paper is organized as follows. In section 2, relevant literature has been reviewed. Essential techniques used in problem formulation and simulation algorithm have been discussed in section 3. In section 4, numerical analysis has been conducted. In section 5, the conclusion and future research have been present .

\section{LITERATURE REVIEW}

\subsection{Supply Chain Risks}

Risk category has been classified into different categories according to different researchers. Tang (2006) has classified the supply chain risk into 4 main parts: supply, demand, product and information. Chopra and Sodhi (2004) have categorized the supply chain risk into 9 areas: 1) disruptions 2) delays 3) systems 4) forecast 5) intellectual property 6) procurement 7) receivables 8) inventory 9) capacity. Here, it has been divided into two groups: inherent and exogenous risks.

Lee et al. (1997) present the bullwhip effect in a supply chain and analyze four sources of the bullwhip effect. Furthermore, Lee et al. (2000) investigate the information sharing in a two-level supply chain with nonstationary end demands. They suggest that the value of demand information sharing can be quite efficient, especially when demands are highly correlated over time. Qi et al. (2004) analyze the supply chain coordination with demand disruption in a deterministic scenario and they show that changes to the original plan induced by a disruption will lead to significant cost across the system. For the supply side disruption, Song and Zipkin (1996) consider the inventory control under supply breakdown by modeling the system as a discrete-time Markov process. Tomlin (2006) studies a single-product case in which a firm can source from two suppliers, one unreliable and one reliable but more expensive. He finds that a supplier's up time and the nature of the disruptions are key factors to determine the optimal strategy. He provides several contingency strategies and compares them in different settings.

The above research is either in discrete time setting, or deterministic scenario, or only considering inherent risk. To the author's knowledge, so far there is little research in supply chain area which quantitatively models supply chain risk as a jump-diffusion process, including both the inherent and exogenous risks.

\subsection{Continuous-Time Inventory Model}

One-product inventory model where the demand follows Wiener process is firstly considered by Bather (1966). Inspired by this paper, a variety of extensions and discussions have followed this direction. Puterman (1975) investigates a continuous-time stochastic storage model which assuming to implement $\left(\mathrm{x}_{0}, \mathrm{x}_{1}\right)$ policy. Harrison and Taylor
(1978) have computed explicitly an optimal policy involving two critical numbers for a Brownian storage system. A review about deterministic and stochastic control theory has been given by Neck (1984). Bar-Lev et al. (1993) analyze the Brownian inventory system with supplier uncertainty by using impulse control. An explicit formula of the expected total discounted cost for an infinite time horizon has been proposed by Dohi et al. (1993). Beyer (1994) presents a special one-product inventory model with Wiener demand process with a fixed positive lead time and a (r, Q) strategy. Duncan et al. (1999) implement adaptive control for stochastic manufacturing systems with hidden Markovian demands and small noise. Since two-number inventory model has been thoroughly studied in literature, it has also been implemented in our study.

\section{MODELING AND SIMULATION ALGORITHM}

The unexpected event usually happens on a sudden and has a tremendous effects on the system. Hence to model the disruption and find an optimal control policy to minimize the effects of the disruption will be meaningful and significant. The inventory level can be modeled as discrete time stochastic process, where it changes only at the end of a day or it can be modeled as continuous stochastic process, where the inventory level movements changes continuously, even though the inventory level is only observed at discrete time points. One can think the inventory level denotes the 'true position' of the inventory which always exists and is varying according to time. Another advantage of continuous time stochastic process is to accurately capture the time when jumps happen and better describe the process evolution. At the mean time, it can also avoid some problems dealing with integration and differentiation in discrete time. Therefore, in our study the inventory level has been set to follow a continuous-time stochastic process.

In the following sections, we present several relevant components of jump-diffusion model. Firstly, Wiener process and its generalized form have been introduced. Secondly, the zero-one jump law used to generate jumps in the simulation is present. Thirdly, the Laplace distribution which is used to describe the jump distribution has been shown next. Lastly, problem formulation and simulated annealing have been discussed.

\subsection{Wiener Process}

In discrete-time time series model, the shocks are assumed to form a white noise process, which are not predictable. The counterpart of shocks in a continuous-time model is the increments of a Wiener process, which is also called a standard Brownian motion. If we focus on small change $\Delta w_{t}=w_{t+\Delta t}-w_{t}$ associated with a small increment $\Delta t$ in time, then a continuous time stochastic process $\left\{w_{t}\right\}$ is a 


\section{Chen and Zhang}

Wiener process if it satisfies:1) $\Delta w_{t}=\varepsilon \sqrt{\Delta t}$, where $\varepsilon$ is a standard normal random variable, which indicates $\left.\Delta w_{t} \sim N(0, \Delta t) ; 2\right) \Delta w_{t}$ is independent of $w_{j}$ for all $j \leq t$.

\subsection{Generalized Wiener Process}

The Wiener process is a special stochastic process with 0 drift and variance proportional to the length of time interval, which means that the rate of change in expectation is 0 and the rate of change in variance is 1 . The generalized Wiener process is such that the expectation has a drift rate $\mu$ and the rate of variance change is $\sigma^{2}$. Denote the generalized Wiener process by $x_{t}$ and use the notation dy for a small change in variable $\mathrm{y}$. Then the generalized Wiener process for $x_{t}$ is $d x_{t}=\mu d t+\sigma d w_{t}$, where $w_{t}$ is a Wiener process.

\subsection{Zero-One Jump Law}

According to Hanson (2007), the jump process should follow the zero-one jump law which will be later implemented in the simulation program. Let $\mathrm{dt}>0$ and $\lambda$ be positive and bounded, then we have

1) $\operatorname{prob}[d P(t)=0]=1-\lambda d t$

2) $\operatorname{prob}[d P(t)=1]=\lambda d t$;

3) $\operatorname{prob}[d P(t)>1]=0$;

where $\mathrm{P}(\mathrm{t})$ denotes the jump process which has a fixed jump rate $\lambda$ and $\mathrm{dP}(\mathrm{t})$ represents the differential in $\mathrm{P}(\mathrm{t})$ and it implies that any jump will be captured in $\mathrm{dP}(\mathrm{t})$. The first two properties represent that as the jump rate increases, the probability of occurring a jump increases accordingly. Property (3) implies that in any particular $\mathrm{dP}(\mathrm{t})$ the number of jumps will not exceed 1 . These rules will be implemented in the simulation study when introducing a jump.

\subsection{Laplace Distribution}

In reality, the jump size won't be the same over time, hence one way to model the jump as following certain distributions, i.e. Laplace distribution which has been used in Kou (2002) and has some nice property, i.e. leptokurtic. The Laplace distribution with pdf

$$
f(x)=\frac{1}{2 b} e^{-\left|\frac{x-\mu}{b}\right|},
$$

where $\mu$ is the location parameter and $b$ is the scale parameter. And its cdf is as follow,

$$
F(x)=\int_{-\infty}^{\infty} f(t) d t=\int_{-\infty}^{\infty} \frac{1}{2 b} e^{-\left|\frac{x-\mu}{b}\right|} d t
$$

$$
\begin{gathered}
= \begin{cases}\frac{1}{2} e^{-\frac{\mu-x}{b}} & \text { if } \mathrm{x}<\mu \\
1-\frac{1}{2} e^{-\frac{x-\mu}{b}} & \text { if } \mathrm{x} \geq \mu\end{cases} \\
=0.5\left[1+\operatorname{sgn}(x-\mu)\left(1-\exp \left(-\frac{|x-\mu|}{b}\right)\right)\right],
\end{gathered}
$$

where sgn is denoted as sign function. Now, consider to find the inverse of the cumulative function, let $p=F(x)$, then we want to express $\mathrm{x}$ as a function of $\mathrm{p}$.

$$
\Rightarrow 2 *(p-0.5)=\operatorname{sgn}(x-\mu)\left(1-\exp \left(-\frac{|x-\mu|}{b}\right)\right),
$$

if $x \geq \mu$, then

$$
\Rightarrow x=\mu-b^{*} \ln (1-2 *|p-0.5|) \text { if } p>0.5,
$$

if $x<\mu$, then

$$
\Rightarrow x=\mu+b^{*} \ln (1-2 *|p-0.5|) \text { if } p<0.5,
$$

therefore, we have

$$
x=\mu-\operatorname{sgn}(p-0.5) * b * \ln (1-2 *|p-0.5|),
$$

and from $1-2 *|\mathrm{p}-0.5|>0$, we have $-0.5<\mathrm{p}-0.5$ : $=\mathrm{U}<$ 0.5 . Hence, given a random variable $U$ drawn from the uniform distribution in the interval $(-0.5,0.5)$, the variable $\mathrm{x}=$ $\mu-\operatorname{sgn}(U) * b * \ln (1-2|U|)$ has a Laplace distribution with parameters $u$ and $b$. This algorithm will be used to generate Laplace distribution. The plot of Laplace distribution has been drawn in Figure 1, which shows that at $m u=0$, it reaches the peak value. And the location parameter mu won't affect the scale value of the function, so in our simulation study, scale parameter is our main interest in order to generate different jump sizes.

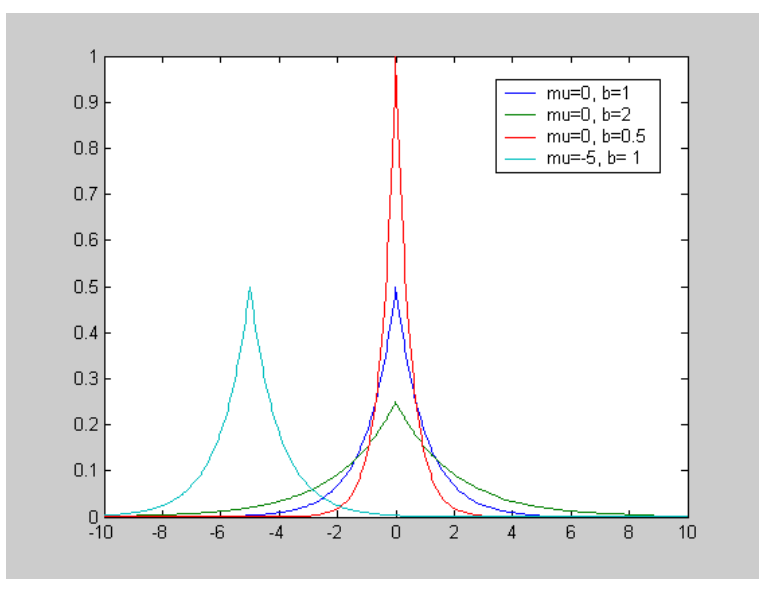

Figure 1: Laplace distribution plot. 


\section{Chen and Zhang}

\subsection{Problem Formulation}

Consider the demand following a jump-diffusion model

$$
d D_{t}=\mu d t-\sigma d w_{t}-d J,
$$

where $D_{t}$ is the demand at time $t$ and notations for other variables are shown in Table 1 . The problem is to minimize the total cost including inventory holding cost, back$\log$ cost (i.e. the inventory level $<0$ ), production cost and machine switching cost.

Table1: Variable notations.

\begin{tabular}{|c|c|}
\hline $\mathrm{X}_{\mathrm{t}}$ & the inventory level at time $\mathrm{t}$ \\
\hline q & the production rate at time $\mathrm{t}$ ( $=1$ in our study) \\
\hline$\mu$ & the demand rate at time $\mathrm{t}$ \\
\hline$\sigma^{2}$ & the variance in demand per unit time \\
\hline$w_{t}$ & the standard wiener process \\
\hline$p$ & the unit backlog penalty cost \\
\hline $\mathrm{h}$ & the unit holding cost ( $=1$ in our study) \\
\hline $\mathrm{c}$ & $\begin{array}{l}\text { the production cost coefficient }(c=0 \text {, since it } \\
\text { won't affect the final result according to Vick- } \\
\text { son }(1986))\end{array}$ \\
\hline $\mathrm{k}$ & the switching cost per time \\
\hline $\mathrm{T}$ & the time length \\
\hline $\mathrm{X}_{0}$ & the initial inventory level ( $=0$ in our study) \\
\hline $\mathrm{S}\left(\mathrm{x}_{\mathrm{t}}\right)$ & the average switching cost \\
\hline $\mathrm{P}\left(\mathrm{x}_{\mathrm{t}}\right)$ & production cost rate at time $\mathrm{t}(=0$, since $\mathrm{c}=0)$ \\
\hline $\mathrm{C}\left(\mathrm{x}_{\mathrm{t}}\right)$ & inventory/penalty cost rate at time $\mathrm{t}$ \\
\hline mode(i) & the machine state at state $\mathrm{i}$ \\
\hline $\mathrm{J}$ & $\begin{array}{l}\text { the compound Poisson process with intensity } \\
\lambda \text { and magnitude } v\end{array}$ \\
\hline $\mathrm{g}$ & the long-term average cost \\
\hline
\end{tabular}

The objective function is

$$
\min E\left\{\int_{0}^{T}\left(C\left(x_{t}\right)+P\left(x_{t}\right)\right) d t+S\left(x_{t}\right)\right\},
$$

where $C\left(x_{t}\right)=\left\{\begin{array}{ll}-p x_{t} & \text { if } x_{t}<0 \\ h x_{t} & \text { if } x_{t}>0\end{array}\right.$,

and the inventory level follows

$$
\begin{aligned}
d x_{t} & =I(\bmod e(i)>0) q d t-d D_{t} \\
& =(I(\bmod e(i)>0) q-\mu) d t+\sigma d w_{t}+d J
\end{aligned}
$$

with $x_{0}=X_{0}$, where I(.) is an indicator function when the insider condition is true, the value is 1 , otherwise 0 ; mode( $i)$ is the machine state, $\operatorname{mode}(i)=1$ when the machine is on at state $i$; when the machine is off at state $i, \operatorname{mode}(i)=$ 0 . This model consists of two parts, with the first part being diffusion process and the second part being a jump process. The occurrences of jumps are governed by a Pois- son process, and the jump size can be constant or follows a certain distribution, i.e. Laplace distribution.

\subsection{Simulated Annealing}

Vickson (1986) use Newton's method, see Kincaid and Cheney (2001), to find the optimal critical levels of $\left(\mathrm{x}_{0}, \mathrm{x}_{1}\right)$ for only diffusion scenario. The model he considered does not include the jump process component, in other words, the optimal values will not be optimal if there is a jump risk in the supply chain. In this paper we use Simulated Annealing (SA) method to find the critical values for the jump-process model, furthermore, we'll analyze the outcomes from the simulation studies.

Simulated annealing (SA) is a random-search method which is analogous as the way a metal cools and freezes into a minimum energy crystalline structure - that is the annealing process - and search for a minimum in a more general system. The major advantage of SA over other methods is the ability to avoid becoming trapped in local minima. The original idea is proposed by Kirkpatrick et al. (1983), who develop the similarities between statistical mechanics and combinatorial optimization, and then apply to a number of problems in optimal design of computers.

The upward moves are accepted with a probability determined by Metropolis function $\left(e^{-\Delta C / T}\right)$, where $\Delta C$ denotes the cost difference of moves. As $\mathrm{T}$ decreases, the probability of acceptance of upward move decreases.

The initial temperature need to be high so that it could find the global optimal solutions, but not too high since it will lead to a long processing time. The initial temperature can be estimated by using the procedure in Wong and Liu (1986). The Metropolis function $\left(e^{-\Delta C / T}\right)$ is used to determine the initial value of $\mathrm{T}$.

The average cost difference is given as

$$
\Delta C_{\mu}=\sum_{i=1}^{M_{\mu}} \Delta C_{i} / M_{\mu}
$$

where $\mathrm{M}_{\mathrm{u}}$ denotes the number of upward moves. Hence, the initial value of $\mathrm{T}$ can be estimated as

$$
T_{0}=\left|\frac{\Delta C_{\mu}}{\ln \left(P_{0}\right)}\right|,
$$

where $P_{0} \approx 0.5$, since we wish to accept the upward moves and downward moves with the same probability, i.e. 0.5.

The simulated annealing algorithm is implemented as follows:

1) Choose a random design vector $X_{i}=\left(x_{0}^{i}, x_{1}^{i}\right)$, where $\left(x_{0}^{i}, x_{1}^{i}\right)$ refers to the two-number inventory 


\section{Chen and Zhang}

policy at state i. Select the initial temperature, and then specify the annealing schedule, i.e. temperature reduction ratio, number of iterations before temperature reduction, etc.

2) Evaluate $\mathrm{F}\left(\mathrm{X}_{\mathrm{i}}\right)$ by a simulation model, where $\mathrm{F}\left(\mathrm{X}_{\mathrm{i}}\right)$ denotes the objective function value when the design vector is $\mathrm{X}_{\mathrm{i}}$

3) Adjust step length to obtain a new neighboring design vector $\mathrm{X}_{\mathrm{i}+1}$

4) Evaluate $\mathrm{F}\left(\mathrm{X}_{\mathrm{i}+1}\right)$ by a simulation model

5) If $\mathrm{F}\left(\mathrm{X}_{\mathrm{i}+1}\right)<\mathrm{F}\left(\mathrm{X}_{\mathrm{i}}\right)$, i.e. $F\left(X_{i+1}\right)-F\left(X_{i}\right)=\Delta C<0$, then $X_{\mathrm{i}+1}$ is the new current solution

6) If $F\left(X_{i+1}\right)>F\left(X_{i}\right)$, then accept $X_{i+1}$ as the new current solution with probability $e^{-\Delta C / T}$

7) Reduce the temperature according to the annealing schedule

8) Terminate the algorithm if satisfying the terminating criteria

where $F\left(X_{i}\right)=\sum_{j=1}^{N} V_{j}\left(X_{i}\right) / N, \mathrm{~V}_{\mathrm{j}}$ is the total cost at sampling $\mathrm{j}$ for design vector $\mathrm{X}_{\mathrm{i}}, \mathrm{N}$ is the number of samplings.

\section{NUMERICAL STUDY}

\subsection{Preliminary Numerical Study}

In order to investigate the effects of the risk, the simulation parameters have been tuned in order to match the examples in Vickson (1986). After several times adjustments based on the numerical results by observing the function cost different $\Delta C$, the initial temperature has been chosen to be 20 in our simulation. The parameter of temperature reduction ratio has been set as 0.8 in our simulation, the number of iterations before temperature reduction has been set to be 10 , and the termination criteria has been set less than $1 \mathrm{e}-3$.
The results have been shown below in Table 2, it can be seen that this setting has a very close results (less than $5 \%$ deviation) as the results in Vickson (1986).

Example 11 in Table 2 has been selected in our later simulation study, the variance of the process can be computed as $\sigma^{2} \Delta t=1.8 *(4500 / 10000)=0.81$, where parameters 4500 and 10000 are the time length and sampling points in our simulation. And we set the intensity of the Poisson process to be $1 \%$, that is, if the time is 4500 units, then there will be 45 times of jumps during the period, which is consistent with the reality, the disruption rarely occurs. The simulation has been conducted to find the average cost $\mathrm{g}$ for different constant jump sizes by using the same $\mathrm{x}_{0}$ and $\mathrm{x}_{1}$ in Vickson (1986). And the results are shown in Table 3.

From Table 3, it is noted that the value of $\mathrm{g}$ is not always increasing as incorporating jumps into the process. An interpretation would be that considering a case at time $\mathrm{t}$, in which the machine is off and inventory level is very close to $\mathrm{x}_{0}$, if the diffusion part $\mathrm{dw}_{\mathrm{t}+1}$ happens to be negative noise at time $\mathrm{t}+1$, and then the inventory level will drop below the critical point $\mathrm{x}_{0}$, without integrating positive jumps, and this will lead to a machine switching cost and backlog inventory cost, recalling the two-number inventory policy in section 1 . If at that particular point, a proper positive jump occurs which will compensate the negative diffusion and keep the inventory level stay in the normal level and it won't cause switch and average cost. Hence, the value of $g$ is not necessarily to be monotonic, in other words a jump with right time, right direction, and right magnitude might reduce the long-term average cost.

Another interesting phenomenon is that for this particular setting, the negative jumps will deteriorate the production process more than the positive jumps. A rational explanation for this case is that the production rate is 1 , and the demand rate is 0.9 that are very close to the production

Table 2: SA algorithm parameter tuning results.

\begin{tabular}{ccccccccccc}
\hline & & & & & \multicolumn{3}{c}{ Vickson's Result } & \multicolumn{3}{c}{ Search $\mathrm{x}_{0}$ and $\mathrm{x}_{1}$ by SA } \\
\cline { 7 - 11 } Ex. & $\mu$ & $\sigma^{2}$ & $\mathrm{p}$ & $\mathrm{k}$ & $\mathrm{x}_{0}$ & $\mathrm{x}_{1}$ & $\mathrm{~g}$ & $\mathrm{x}_{0}$ & $\mathrm{x}_{1}$ & $\mathrm{~g}$ \\
\hline 1 & 0.5 & 1.0 & 2 & 25 & -1.57 & 3.39 & 3.45 & -1.14 & 3.35 & 3.64 \\
2 & 0.5 & 1.0 & 2 & 50 & -2.18 & 4.50 & 4.52 & -2.15 & 4.95 & 4.83 \\
3 & 0.5 & 1.0 & 6 & 25 & -0.30 & 4.32 & 4.38 & -0.23 & 4.55 & 4.56 \\
4 & 0.5 & 1.0 & 6 & 50 & -0.60 & 5.53 & 5.54 & -0.58 & 5.42 & 5.89 \\
5 & 0.5 & 1.0 & 10 & 25 & 0.17 & 4.79 & 4.83 & 0.24 & 4.48 & 4.99 \\
6 & 0.5 & 1.0 & 10 & 50 & -0.10 & 5.98 & 5.99 & -0.08 & 6.29 & 6.27 \\
7 & 0.9 & 1.8 & 2 & 25 & 5.99 & 12.25 & 10.48 & 5.56 & 12.78 & 10.73 \\
8 & 0.9 & 1.8 & 2 & 50 & 5.28 & 13.17 & 10.80 & 5.55 & 12.79 & 11.01 \\
9 & 0.9 & 1.8 & 6 & 25 & 13.62 & 19.87 & 18.11 & 13.95 & 21.18 & 18.72 \\
10 & 0.9 & 1.8 & 6 & 50 & 12.90 & 20.80 & 18.42 & 13.15 & 22.38 & 19.00 \\
11 & 0.9 & 1.8 & 10 & 25 & 17.68 & 23.94 & 22.17 & 18.75 & 23.98 & 22.43 \\
12 & 0.9 & 1.8 & 10 & 50 & 16.97 & 24.87 & 22.49 & 18.15 & 25.38 & 22.79 \\
\hline
\end{tabular}




\section{Chen and Zhang}

rate, which means the accumulation of inventory is slow. While the initial inventory level has been set at 0 , the positive jump will make more contribution to help accumulate the inventory level between $x_{0}$ and $x_{1}$ in order to keep the production process stable. On the other hand, the negative jumps will be much easier to disrupt the production and lead to switching cost and backlog cost.

Furthermore, note that the backlog cost $\mathrm{p}$ is 10 , and the holding cost $\mathrm{h}$ is 1 , so the backlog cost is 10 times as holding cost and the switching cost $\mathrm{k}$ is 25 , which is even more expensive. Due to slow inventory accumulation and the higher cost of switching and backlog compared to holding, that is why the negative jumps lead to much higher value of $g$ than positive jumps.

Table 3: The total cost with constant jumps in inventory.

\begin{tabular}{cccc}
\hline Case & $\mathrm{v}$ & $\begin{array}{c}\% \text { of } \\
\text { variance }\end{array}$ & $\mathrm{g}$ \\
\hline 1 & 0 & No Jump & 21.97 \\
2 & 0.1 & $12.35 \%$ & 19.27 \\
3 & 0.2 & $24.69 \%$ & 21.10 \\
4 & 0.3 & $37.04 \%$ & 23.48 \\
5 & 0.4 & $49.38 \%$ & 56.57 \\
6 & 0.5 & $61.73 \%$ & 498.47 \\
7 & -.005 & $-0.6 \%$ & 25.18 \\
8 & -0.01 & $-1.23 \%$ & 31.79 \\
9 & -0.03 & $-3.70 \%$ & 102.84 \\
10 & -0.05 & $-6.17 \%$ & 478.65 \\
\hline
\end{tabular}

\subsection{Constant Jump-Diffusion Process}

In order to demonstrate the process, the example 11 in Table 2 has been used in simulation study where the critical points $\mathrm{x}_{0}$ and $\mathrm{x}_{1}$ have been obtained by simulated annealing algorithm and the optimal long-term average $g$ has been compared to the previous simulation result where the $\mathrm{x}_{0}$ and $\mathrm{x}_{1}$ have been set to be constant.

In this simulation, the jumps are set as a constant. The simulation results are shown in Table 4 . Recall that the demand has been modeled as a jump-diffusion

$$
d D_{t}=\mu d t-\sigma d w_{t}-d J
$$

and the inventory level follows

$$
\begin{aligned}
d x_{t} & =I(\bmod e(i)>0) q d t-d D_{t} \\
& =(I(\bmod e(i)>0) q-\mu) d t+\sigma d w_{t}+d J
\end{aligned}
$$

where $D_{t}$ is the demand at time t. Since the supply chain is considered as one-echelon, so the jump direction is opposite between demand and inventory level, that is, the jump direction in table 3 and table 4 is opposite. The reason to present in this way is to give more intuitive interpretation for later simulation results.

It is noted that as a positive jump occurs in demand, the value of $g$ increases. And the larger size of jump, the larger interval between $\mathrm{x}_{0}$ and $\mathrm{x}_{1}$. It can be explained as to minimize the average cost, the critical points should be set further away between each other to contain more variation in demand, keep the production stable and avoid switch cost. An interesting phenomenon is when a negative jump occurs in demand, those jumps can be seen as benefits to the production process since it reduces the average cost $\mathrm{g}$ in comparison with that in fixed $x_{0}$ and $x_{1}$. One way to explain this is that when the inventory accumulates very close to $\mathrm{x}_{1}$, if without a negative jump, then it will lead to switch cost, so a negative jump compensates the inventory level and keep the production stable in the meantime it reduces the switch costs. From another perspective, as the negative jump size is increasing, the interval between $\mathrm{x}_{0}$ and $\mathrm{x}_{1}$ is decreasing, which implies that the production process doesn't need large interval between $\mathrm{x}_{0}$ and $\mathrm{x}_{1}$ to

\begin{tabular}{|c|c|c|c|c|c|c|c|c|}
\hline \multirow[b]{2}{*}{ Case } & \multirow[b]{2}{*}{$\begin{array}{l}\text { Jump in } \\
\text { Demand }\end{array}$} & \multirow[b]{2}{*}{$\begin{array}{l}\% \text { of va- } \\
\text { riance }\end{array}$} & \multicolumn{3}{|c|}{$\begin{array}{l}\text { Simulation based on constant } x_{0} \\
\text { and } x_{1}\end{array}$} & \multicolumn{3}{|c|}{$\begin{array}{l}\text { Search } \mathrm{x}_{0} \text { and } \mathrm{x}_{1} \text { by } \\
\text { SA algorithm }\end{array}$} \\
\hline & & & $\mathrm{x}_{0}$ & $\mathrm{x}_{1}$ & $\mathrm{~g}$ & $\mathrm{x}_{0}$ & $\mathrm{X}_{1}$ & $\mathrm{~g}$ \\
\hline 1 & 0 & No Jump & 17.68 & 23.94 & 21.97 & 18.75 & 25.98 & 22.43 \\
\hline 2 & -0.1 & $-12.35 \%$ & 17.68 & 23.94 & 19.27 & 4.33 & 13.12 & 11.72 \\
\hline 3 & -0.2 & $-24.69 \%$ & 17.68 & 23.94 & 21.10 & 2.32 & 6.46 & 7.73 \\
\hline 4 & -0.3 & $-37.04 \%$ & 17.68 & 23.94 & 23.48 & 0.98 & 5.04 & 6.38 \\
\hline 5 & -0.4 & $-49.38 \%$ & 17.68 & 23.94 & 56.57 & 0.41 & 3.51 & 6.71 \\
\hline 6 & -0.5 & $-61.73 \%$ & 17.68 & 23.94 & 498.47 & -0.91 & 0.82 & 12.00 \\
\hline 7 & 0.005 & $0.6 \%$ & 17.68 & 23.94 & 25.18 & 13.20 & 28.95 & 21.51 \\
\hline 8 & 0.01 & $1.23 \%$ & 17.68 & 23.94 & 31.79 & 17.75 & 38.27 & 21.61 \\
\hline 9 & 0.03 & $3.70 \%$ & 17.68 & 23.94 & 102.84 & 18.65 & 56.32 & 26.36 \\
\hline 10 & 0.05 & $6.17 \%$ & 17.68 & 23.94 & 478.65 & 23.70 & 66.88 & 67.35 \\
\hline
\end{tabular}
keep stable. In other words, there is little variation in the

Table 4: Results for jump-diffusion processes with constant jumps in demand. 


\section{Chen and Zhang}

demand process, which furthermore justifies the benefits of negative jumps to the production process.

And these results confirm to the intuition that the larger demand, the more inventory; the less demand, the less inventory.

\subsection{Jumps Following Laplace Distribution}

In previous study, the magnitude of jumps is assumed to be constant, while the simulation for the magnitude of jumps following certain distribution, i.e. Laplace distribution, has been conducted in this section, since it has several favorable properties, such as leptokurtic feature, memoryless property, see Kou (2002). So in our simulation, the jumps which follow Laplace distribution have been integrated with the diffusion process and the simulation results are shown in Table 5.

From Table 5, it is noted that when the scale parameter $b=10,000$, the maximum jump size is $1 /(2 * 10,000)=$ $5 \mathrm{e}-5$, which implies that the result is very close to the no jump case. And it also shows that in overall the long-term average cost $g$ has been reduced by using SA algorithm and it has a similar simulation result as constant jumps, but the assumption of jump sizes following Laplace distribution is much closer to reality.

\subsection{Cost Structure for Jump-Diffusion Processes}

To provide a snapshot of the simulation process and better understand the cost structure, the simulation random generator has been chosen to a certain state, that is, the time series is generated as the same regardless how many times the program runs. In our study, the generator state has been set to 20 . And the inventory and backlog costs have been shown in two independent parts. The simulation results are summarized in Table 6.

From Table 6, it can be seen that inventory and back$\log$ costs are the main part of total cost compared with switching cost. From case 2 to 6 , it is shown that when there is a negative jump on the demand, the holding cost is increasing $(>12.96)$ compared with the holding cost of no jump since more inventory will accumulate when the demand drops if the production rate keeps the same. And it shows that the holding cost occupies most portion of total cost. From case 7 to 10, when there is a positive jump on the demand, and the production rate maintains the same, the results show that the backlog cost has a significant increase, especially when the maximum jump size is larger than $3 \%$ of the variance of the process.

The outputs shows that when the production rate is close to the demand rate, and when the backlog penalty is much larger than holding cost, a positive jump on demand has more serious effects than a negative jump since it will lead to more backlog costs. Note that when the parameters change, i.e. production rate, demand rate, the results may change accordingly. In our study, a specific parameter setting has been used to illustrate the effects of jumps on the performance of supply chain.

\section{CONCLUSION AND FUTURE RESEARCH}

This paper mainly investigates the effects of jumps occurred in a diffusion process. First of all, jump-diffusion modeling techniques have been introduced. Then Simulated Annealing (SA) algorithm has been used to find the optimal critical values for two-number inventory policy $\left(\mathrm{x}_{0}, \mathrm{x}_{1}\right)$ when demand disruptions occur and it shows that the long-term average cost has been reduced. Numerical studies have been conducted to study the jump-diffusion model. From the simulation results, it has shown that not all jumps are harmful to the process; in some cases a jump with a proper magnitude and appropriate direction probably will bring benefits to the system.

Note that the optimality conditions in Vickson (1986) are only sufficient, which means that the two-number inventory policy is not unique optimal policy. Hence there

Table 5: Results for jump-diffusion processes with jumps from Laplace distribution.

\begin{tabular}{cccccc}
\hline Case & $\begin{array}{l}\text { Scale Parameter } \\
\text { of Lap. Dist. b/-b }\end{array}$ & $\begin{array}{l}\text { Max. Jump size } \\
\text { in Demand }\end{array}$ & \% of variance & $\begin{array}{l}\text { Constant } \mathrm{x}_{0} \text { and } \\
\mathrm{x}_{1} \text { for g }\end{array}$ & $\begin{array}{l}\text { Laplace Jump } \\
\text { size for } \mathrm{g}\end{array}$ \\
\hline 1 & 10,000 & 0 & No Jump & 21.97 & 22.46 \\
2 & 5 & -0.1 & $-12.35 \%$ & 19.27 & 18.42 \\
3 & 2.5 & -0.2 & $-24.69 \%$ & 21.10 & 19.89 \\
4 & 1.67 & -0.3 & $-37.04 \%$ & 23.48 & 20.94 \\
5 & 1.25 & -0.4 & $-49.38 \%$ & 56.57 & 21.80 \\
6 & 1 & -0.5 & $-61.73 \%$ & 498.47 & 22.69 \\
7 & -100 & 0.005 & $0.6 \%$ & 25.18 & 24.11 \\
8 & -50 & 0.01 & $1.23 \%$ & 31.79 & 25.65 \\
9 & -16.67 & 0.03 & $3.70 \%$ & 102.84 & 36.97 \\
10 & -10 & 0.05 & $6.17 \%$ & 478.65 & 69.83 \\
\hline
\end{tabular}


Chen and Zhang

Table 6: Cost structure of jump-diffusion process.

\begin{tabular}{lccccccc}
\hline \multirow{2}{*}{$\begin{array}{c}\text { Case } \\
\text { variance }\end{array}$} & $\begin{array}{c}\text { \%witch } \\
\text { times }\end{array}$ & $\begin{array}{c}\text { Switch } \\
\text { cost }\end{array}$ & $\begin{array}{c}\text { Holding } \\
\text { cost }\end{array}$ & $\begin{array}{c}\text { Backlog } \\
\text { cost }\end{array}$ & $\begin{array}{c}\text { Total cost of } \\
\text { Inv. \& back- } \\
\text { log }\end{array}$ & $\begin{array}{c}\text { Total } \\
\text { cost }\end{array}$ \\
\hline 1 & No Jump & 96 & 0.24 & 12.96 & 12.51 & 25.47 & 25.71 \\
2 & $-12.35 \%$ & 194 & 0.49 & 17.14 & 0.91 & 18.05 & 18.54 \\
3 & $-24.69 \%$ & 269 & 0.67 & 18.70 & 0.07 & 18.76 & 19.45 \\
4 & $-37.04 \%$ & 296 & 0.74 & 19.92 & 0.04 & 19.95 & 20.69 \\
5 & $-49.38 \%$ & 310 & 0.78 & 20.87 & 0.02 & 20.89 & 21.66 \\
6 & $-61.73 \%$ & 286 & 0.72 & 21.94 & 0.01 & 21.95 & 22.67 \\
7 & $0.6 \%$ & 90 & 0.23 & 12.62 & 14.15 & 26.76 & 26.99 \\
8 & $1.23 \%$ & 88 & 0.22 & 12.36 & 14.95 & 27.31 & 27.53 \\
9 & $3.70 \%$ & 64 & 0.16 & 9.75 & 28.28 & 38.03 & 38.19 \\
10 & $6.17 \%$ & 44 & 0.11 & 6.76 & 62.88 & 69.63 & 69.73
\end{tabular}

may exist other optimal policies and to find such policies and compare them would be an interesting future direction. And in our study the production rate is constant, which could also be considered as a flexible variable. Thus, if considering production rate as a control variable, we could formulate this problem as a stochastic optimal control problem. If the supply chain has been expanded to multiechelon, then it would be another topic to investigate the cost spread among partners in the supply chain.

\section{REFERENCES}

Bar-Lev, S. K., M. Parlar, and D. Perry. 1993. Impulse control of a brownian inventory system with supplier uncertainty. Stochastic Analysis and Application 11: 11-27.

Banks, J., J. S. Carson, B. L. Nelson, and D. M. Nicol. 2000. Discrete-event system simulation. 3rd ed. Upper Saddle River, New Jersey: Prentice-Hall, Inc.

Bather, J. A. 1966. A continuous time inventory model. Journal of Applied Probability 3: 538-549.

Beyer, D. 1994. An inventory model with wiener demand process and positive lead time. Optimization 29: 181193.

Chopra, S., and M. S. Sodhi. 2004. Managing risk to avoid supply-chain breakdown. MIT Solan Management Review 46: 53-61.

Dohi, T., N. Kaio, and S. Osaki. 1993. Continuous time inventory control for wiener proces demand. Computers Mathematical Application 26: 11-22.

Duncan, T. E., B. Parsik-Duncan, and Q. Zhang. 1999. Adaptive control of stochastic manufacturing systems with hidden markovian demands and small noise. IEEE transactions on Automatic Control 44: 427-430.

Graves, S. C., and J. Keilson. 1981. The compensation method applied to a one-product production/inventory problem. Mathematics of Operations Research 6: 246262.

Hanson, F. B. 2007. Applied stochastic process and control for jump-diffusions: modeling, analysis and computation. s.l. : SIAM.

Harrison, J. M., and A. J. Taylor. 1978. Optimal control of a brownian storage system. stochastic Processes and Their Applications 6: 179-194.

Harrison, J. M. 1985. Brownian motion and stochastic flow systems. New York: John Wiley \& Sons, Inc.

Hendricks, K. B., and V. R. Singhal. 2005. An empirical analysis of the effect of supply chain disruptions on long-run stock price performance and equity risk of the firm. Production and Operations Management 14: $53-68$.

Kincaid, D., and W. Cheney. 2001. Numerical analysis: mathematics of scientific computing. Third Edition. Pacific Grove, CA : Brooks/Cole.

Kirkpatrick, S., C. D. Gelatt, and M. P. Vecchi. 1983. Optimization by simulated annealing. Science 220: 4598.

Kou, S. G. 2002. A jump-diffusion model for option pricing. Management Science 48: 1086-1101.

Kushner, H. 1971. Introduction to stochastic control. New York : Holt, Rinehart and Winston.

Lee, H. L., V. Padmanabhan, and S. Whang. 1997. Information distortion in a supply chain: the bullwhip effect. Management Science 43: 546-558.

Lee, H. L., K. C. So, and C. S. Tang. 2000. The value of information sharing in a two-level supply chain. Management Science 46: 626-643.

Neck, R. 1984. Stochastic control theory and operational research. European Journal of Operational Research 17: 283-301.

Puterman, M. L. 1975. A diffusion process model for a 
storage system. [ed.] M. A. Geisler. Logistics 1: 143159.

Qi, X., J. F. Bard, and G. Yu. 2004. Supply chain

coordination with demand disruptions. Omega 32: 301-312.

Song, J. S., and P. H. Zipkin. 1996. Inventory control with information about supply conditions. Management Science 42: 1409-1419.

Tang, C. S. 2006. Perspectives in supply chain risk management. International Journal of Production Economics 103: 451-488.

Tomlin, B. 2006. On the value of mitigation and contingency strategies for managing supply chain disruption risks. Management Science 52: 636-657.

Vickson, R. 1986. A single product cycling problem under brownian motion demand. Management Science 32: 1336-1345.

Wong, D. C., and C. L. Liu. 1986. A new algorithm for floorplan design. Proc. of the $23^{r d} D A C$ : $101-107$.

\section{AUTHOR BIOGRAPHIES}

XIANZHE CHEN is a Ph.D candidate in transportation and logistic and minor in applied mathematics. He holds masters in industrial engineering and statistics. His research interests include stochastic control, supply chain risks, and large-scale systems modeling and optimization.

Dr. JUN ZHANG is an assistant professor in Industrial and manufacturing engineering at North Dakota State University. She obtained her Ph.D. degree from Purdue University in 2006. Her research interests are supply chain management, simulation optimization and stochastic modeling and methodologies. 